\title{
The Improvement of Watershed Algorithm Accuracy for Image Segmentation Handwritten Numbered Musical Notation
}

\author{
Kartono Pinaryanto \\ Department of Informatics, Faculty of Science and Technology, \\ Sanata Dharma University, Yogyakarta, Indonesia \\ Corresponding Author: kartono@usd.ac.id
}

(Received 13-05-2019; Revised 21-05-2019; Accepted 21-05-2019)

\begin{abstract}
In the implementation of image processing to translate the image of the numbered musical notation into a numerical character requires some initial process that must be passed like image segmentation process. The advantage of successful segmentation process is that it can reduce the failure rate in the object recognition process. Segmentation process determines the success of object recognition process, it takes segmentation algorithm that can perform accurate object separation. The combination segmentation process developed in this research used projection profile algorithm, watershed and non-object filtering. Profile projection algorithm is used to crop the image of the musical horizontally and vertically. The watershed algorithm is used to segment the numerical object of numerical notation generated from the projection profile process. Non-object filtering is a continuation of the watershed algorithm that includes the non-object reduction process and the process of combining objects so that the original object segment will be generated. Based on the results of the research, the accuracy of the segment on watershed segmentation is $99.74 \%$ higher than watershed segmentation without combination of $94.82 \%$.
\end{abstract}




\section{International Journal of Applied Sciences and Smart Technologies}

Volume 1, Issue 1, pages 51-64

ISSN 2655-8564

Keywords: watershed algorithm, profile projection algorithm, non-object

filtering, image number notation musical

\section{Introduction}

Indonesian regional songs are a valuable cultural heritage of Indonesia and have important characteristics in each region. Each regional song provides valuable advice or knowledge to the younger generation. Regional songs in addition to consisting of sounds also have a tone or music in the form of score numbers notation [1] so that we as young people are expected to be able to understand and preserve regional songs. One way to understand folk songs is to learn folk songs, especially on scores of number notations by performing image processing.

In the application of image processing to translate the image number notation into numerical characters requires several initial processes (pre-processing) that must be passed like the image segmentation process. The process of image segmentation is a process to separate one object from another object. One advantage of the success of the segmentation process is that it can reduce the level of failure in the object recognition process. In research [2] a method of combining intensity filtering (high pass filtering and low pass filtering) was developed as image pre-processing for noise reduction and watershed transformation to produce better quality segmentation. The result of combining this method is able to reduce excessive over segmentation. Based on research [3] river segmentation is an important process in the river tracking system using unmanned aerial vehicles. This process greatly determines the success of the river tracking system, this is because the output of image segmentation is an input that determines the outcome of the next process.

Based on the research [4] profile projection segmentation algorithm on the Javanese literary text document image Hamong Tani is a relatively good algorithm for document image segmentation with an accuracy of $84.255 \%$ and standard deviation of $14.093 \%$. Based on the research [5] it was tested on Batak characters and the results of segmentation yielded a percentage value of truth segmentation ranging from $65 \%$ to $87.68 \%$ with a confidence level of $95 \%$. Based on the research [6] which examined the image segmentation of braille documents using the projection profile method. The 


\section{International Journal of Applied Sciences and Smart Technologies}

Volume 1, Issue 1, pages 51-64

ISSN 2655-8564

results of the study were able to distinguish front side dots (Recto) and back side dots (Verso). Based on research [7] which examines the text image segmentation on soil maps using radon transformation based projection profile can be used to cluster text blocks. Based on research [8] which examines intelligent systems with the introduction of numerical music notation (NMRPIS) automatically using Optical Music Recognition (OMR). Based on the results of the experiment the level of introduction of NMRPIS reached $99 \%$ and the performance rate was $99.06 \%$.

The watershed segmentation algorithm is one of the segmentation algorithms based on topographic forms [9]. Based on the research [10] the combination of medical image segmentation algorithms, namely reconstruct gradient, float-point active-image, watershed algorithm and the Grab Cut algorithm that functions to cut the image smoothly. In quality the combination of algorithms produces a good image, so that in the process of analysis and analysis will give better results. Based on research [11] watershed segmentation algorithms on Javanese literary text document images produce many objects, so the results of watershed segmentation are not good with an accuracy of $57.433 \%$ which can increase failure in the object recognition process. The results of watershed segmentation contain many objects and not objects so it is necessary to filter out objects and non-objects by throwing away not objects so that the expected level of accuracy is more accurate. Given the importance of the results of the segmentation process as an initial process of object recognition, a segmentation algorithm is needed that can accurately separate objects.

To correct this problem, it is necessary to create a score segmentation algorithm, number notation on a regional song which is a combination of a watershed algorithm, profile projection and filtering not an object. This research is expected to help attract the interest of the younger generation in preserving Indonesian regional culture and applying combinations can help improve the performance of the proposed algorithm in terms of the accuracy of segmentation results.

\section{Research Methodology}

The research method discusses the design of research methods, implementations and datasets. In the design of the research method, in broad outline, it discusses the flow of 


\section{International Journal of Applied Sciences and Smart Technologies}

Volume 1, Issue 1, pages 51-64

ISSN 2655-8564

the process of watershed segmentation combinations and the process flow segmentation wathershed without a combination. In the implementation phase, it discusses the results of implementation of the watershed segmentation program. For datasets explain the types and examples of images used.

\subsection{Design of Research Methods}

In the research method phase, it discusses the design of the combination segmentation process flow between watershed algorithms, profile projection and filtering rather than objects with watershed algorithmic segmentation without combination. The design of the segmentation process flow is shown in Figure 1.

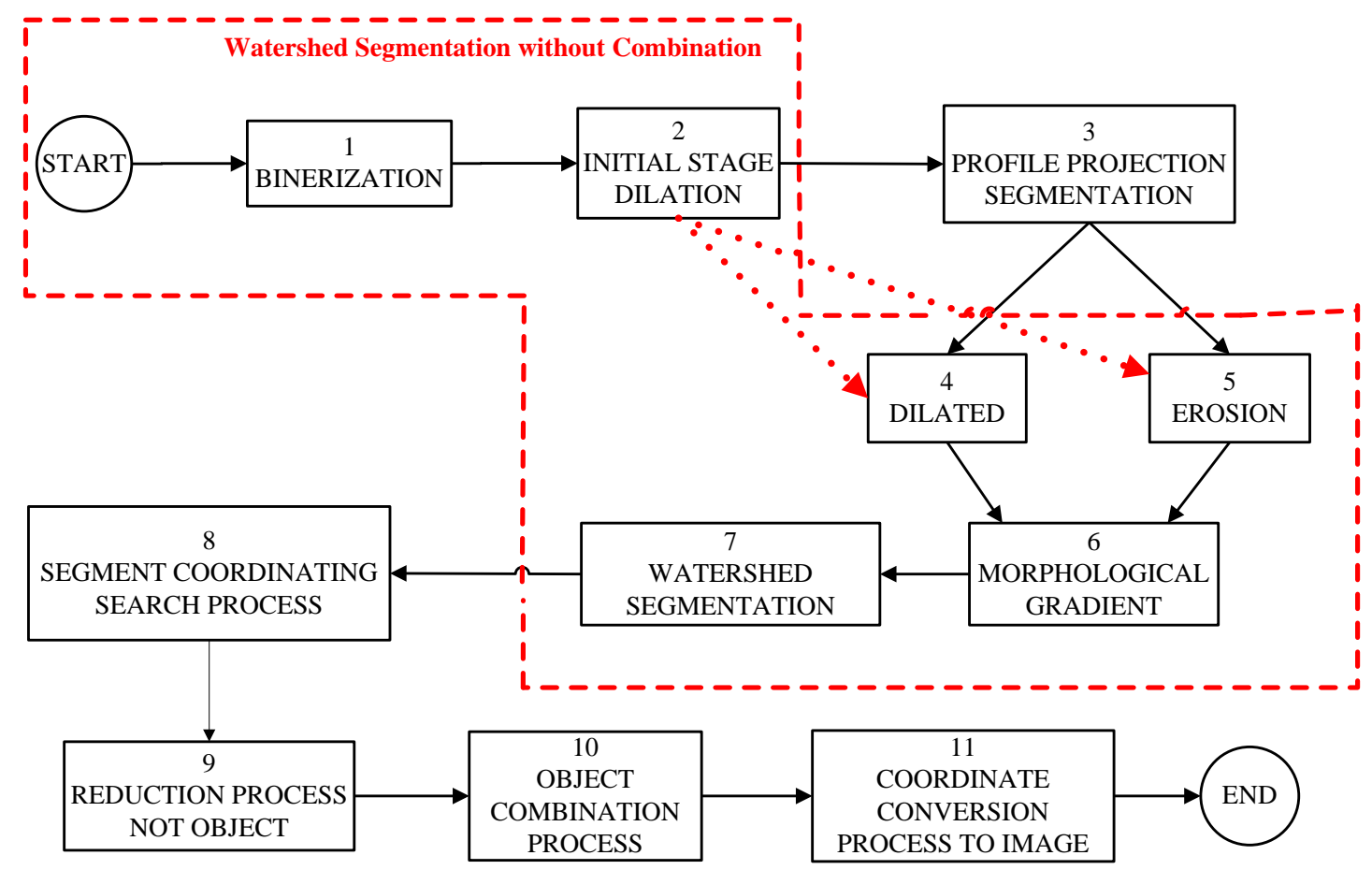

Figure 1. Designing the process flow of the image segmentation number notation

Figure 1 shows the flow of the combined segmentation process from the watershed algorithm, profile projection and filtering not objects that include binaryization processes, dilated early stages until the process of converting coordinates to images, while the design of watershed algorithm segmentation without combination includes 


\section{International Journal of Applied Sciences and Smart Technologies}

Volume 1, Issue 1, pages 51-64

ISSN 2655-8564

binary processes, initial dilation, dilation, erosion, morphological gradient and watershed segmentation.

The binary process is the process of changing the gray scale format into a binary image (black and white). In the binary process, thersholding will be sought, then the point with a certain gray scale value range is changed to black and the rest to white. In this study using the otsu method [9]. The purpose of the otsu method is to divide the gray scale image histogram into two different regions automatically without requiring user assistance to enter the threshold value.

The initial dilation process is the process of enlarging the size of an object and repairing a separate object by adding layers around the object with 8-connected. The purpose of this process is to smooth the binary image so that separate pixels will join into a complete object.

Profile projection is the process of changing a binary image into a single dimension array (histogram) that is perpendicular to the $\mathrm{x}$ axis or $\mathrm{y}$ axis. Image profile projection is divided into 2, namely horizontal profile projections and vertical profile projections. Horizontal profile projection is the number of black pixels perpendicular to the $x$ axis using equation (1):

$$
P_{h}[j]=\sum_{i=1}^{N} S[i, j]
$$

Whereas vertical profile projection is the number of black pixels perpendicular to the $y$ axis using equation (2):

$$
P_{v}[i]=\sum_{j=1}^{M} S[i, j]
$$

Description of equations (1) and (2), namely $S[i, j]$ are images in row $i$ and column $j$, are vertical profile projections, are horizontal profile projections, $M$ is column or image width, $N=$ row or image height, $i$ are indexes for rows $(1,2,3, \ldots, N)$ and $j$ is index for columns $(1,2,3, \ldots, M)$.

Dilation process [9] is a process to increase the size of an object by adding layers around the object with 8-connected. The purpose of the dilation process is slightly different from the initial dilation process, which is to produce a morphological gradient 


\section{International Journal of Applied Sciences and Smart Technologies}

Volume 1, Issue 1, pages 51-64

ISSN 2655-8564

image. The process of erosion [9] is the process of reducing the size of an object by eroding the layer around the object with 8-connected. The purpose of this process is to produce a morphological gradient image. The morphological gradient process [9] is the process by which the new image produced is the result of the difference between the dilation process and the erosion process. The morphological gradient process aims to prevent excessive segmentation. The watershed segmentation process [9] is a regionally based segmentation process that is carried out by dam formation or watershed lines.

Segment coordinate search process is the process of finding the edge of each segment result represented in the form of a row index and segment image column index. The coordinate search process aims as the initial process for reduction rather than objects, combining objects and labeling sequence segments. The segment coordinate search process is carried out using profile projections horizontally and vertically without going through the cutting process. Segment coordinate search results generate a list in the form of a table which includes:

1. A segment number is the result of a numbered segment,

2. The segment line $X 1$ represents the upper edge segment,

3. The segment line Y1 represents the left edge segment,

4. The segment line $\mathrm{X} 2$ represents the lower edge segment, and

5. The segment line Y2 represents the right edge segment

The reduction process is not an object is the process of removing the results of a segment not an object. The reduction process aims to improve the level of accuracy of segmentation. Because the results of segmentation are influenced by the morphological gradient process, the reduction process is divided into 2 stages, namely:

1. The first stage is to reduce not the object in the background segment, and

2. The second stage is to reduce segments not special objects in notations that have holes.

such as number notations 0 and 6 , where each time a segmentation occurs, it will produce segments instead of objects in the form of holes that are considered as object segments.

So a non-object segment is a subset of an object segment so that it can be defined using equation (3): 


\section{International Journal of Applied Sciences and Smart Technologies}

Volume 1, Issue 1, pages 51-64

ISSN 2655-8564

If segment $A \subset$ segment $B$, then delete segment $A$

The process of combining objects is the process of uniting two objects resulting from a segment into a segment object. On the results of watershed segmentation in high notes or low tones 2 segments of objects will be formed so that the object merging process needs to be done. In the point segment and tested segment, a merger process can be carried out if vertically the point segment has 1 area or region with the tested segment, so that it can be defined using equation (4):

If the point segment $\cap$ segment is tested, then join the two segments

The process of converting coordinates to images is the final stage of the segmentation process. The process of coordinating to image is the process of changing the coordinate axis that has undergone a process of reduction rather than an object and the incorporation of objects into segment image files.

\subsection{Implementation}

In the results of the implementation of the combination watershed segmentation program is illustrated in Figure 2.

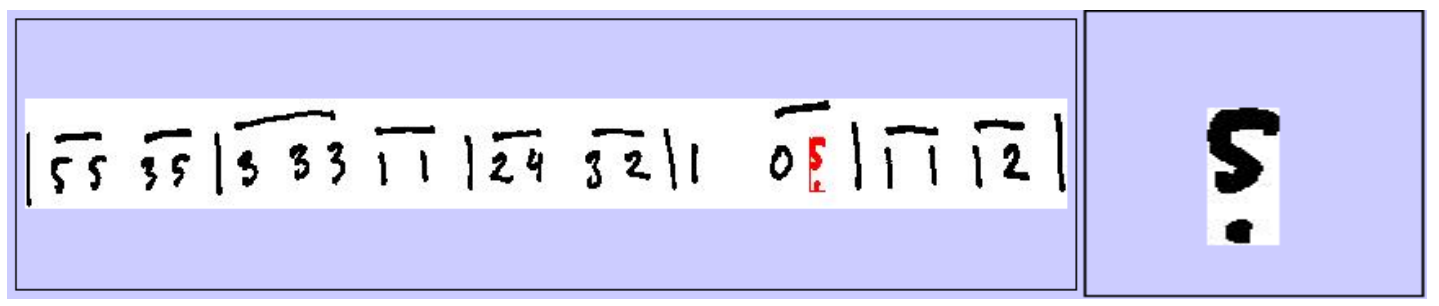

(a)

(b)

Figure 2. Ilustration of segmentation results: (a) Line 1 sub segmentation results. (b) Results of segment image detail 


\section{International Journal of Applied Sciences and Smart Technologies}

Volume 1, Issue 1, pages 51-64

ISSN 2655-8564

\subsection{Dataset}

The dataset used is the image of handwritten number notation on paper (analog data), then the scan process and crop are carried out so that it produces the type of image file jpg (digital data) with a file size of $1800 \times 900$ pixels. Table 1 is a list of datasets that will be used as test data. The category column consists of 3 types of categories, namely simple, medium and complex. In the simple category is a score image that does not have a flat line segment or legato, the medium category is a simple category image and has a 1-level flat line segment or legato, and a complex category is a medium category score image and has a combination of 2-level flat line segments or combinations flat line with legato. Examples of original images of number notation is shown in Figure 3.

Table 1 . Feature image dataset number notation

\begin{tabular}{ccccc}
\hline No & Image name & Title & Origin & Category \\
\hline 1 & Feature image 1 & Cublak cublak suweng & Central Java & Simple \\
2 & Feature image 2 & Naik Naik Ke Puncak Gunung & Maluku & Simple \\
3 & Feature image 3 & Ayo Mama & Maluku & Medium \\
4 & Feature image 4 & Ampar Ampar Pisang & South Borneo & Medium \\
5 & Feature image 5 & Anak Kambing Saya & East Nusa Tenggara Medium \\
6 & Feature image 6 & Gelang Sipaku Gelang & West Sumatra & Medium \\
7 & Feature image 7 & Suwe Ora Jamu & Yogyakarta & Complex \\
8 & Feature image 8 & Burung Tantina & Maluku & Complex \\
9 & Feature image 9 & Yamko Rambe Yamko & Papua & Complex \\
10 & Feature image 10 & Cik Cik Periok & West Borneo & Complex \\
\hline
\end{tabular}

$$
\begin{aligned}
& |\overline{55} \overline{35}| \overline{333} \overline{11}|\overline{24} \overline{32}| 1|\overline{05}| \overline{12} \mid \\
& |\overline{3 \overline{33}} \overline{25}| \overline{33} \overline{22} \mid 1 \text { o } 1 \overline{55} \overline{35}|\overline{3 \overline{33}} \overline{11}| \\
& |\overline{24} \overline{32}| 1 \quad \overline{05}|\overline{11} \overline{12}| \overline{3 \overline{33}} \overline{25}|\overline{33} \overline{22}| \\
& 110551 \overline{11} \overline{12}|\overline{33} \overline{25}| \overline{33} \overline{22}|1 \quad \overline{05}| \\
& |\overline{1 \overline{11}} \overline{12}| 3 \quad \overline{25}|\overline{33} \overline{22}| 1011
\end{aligned}
$$

Figure 3. Original images are handwritten number notations. 


\section{International Journal of Applied Sciences and Smart Technologies}

Volume 1, Issue 1, pages 51-64

ISSN 2655-8564

\section{Results and Discussions}

The results of segmentation testing and discussion were carried out by testing the level of accuracy of the combination watershed and watershed segmentation without combination. Testing the results of watershed segmentation accuracy without a combination only displays segmentation results. In testing the results of the accuracy of segmentation watershed combinations in addition to displaying the results of segmentation, it also displays the results of a non-object reduction process and object merging.

\subsection{Results of Segment Accuracy in Combined Watershed Segmentation}

In Table 2 the results of the accuracy of the watershed segmentation combination with the average test results of the level of accuracy of segments in combination watershed segmentation is $99.74 \%$. The factor that causes the level of accuracy of the segment not to reach $100 \%$ occurs in the 4th image which experiences errors of 6 objects.

Table 2. Results of Combined Watershed Segmentation Accuracy

\begin{tabular}{cccccccc}
\hline \multirow{2}{*}{$\begin{array}{c}\text { Feature } \\
\text { image }\end{array}$} & $\begin{array}{c}\text { Original } \\
\text { image }\end{array}$ & $\begin{array}{c}\text { Segmentation } \\
\text { results image }\end{array}$ & $\begin{array}{c}\text { Reduction } \\
\text { Not Object }\end{array}$ & $\begin{array}{c}\text { Object } \\
\text { Merging }\end{array}$ & True & False & $\begin{array}{c}\text { Accuracy } \\
(\%)\end{array}$ \\
\hline 1 & 79 & 105 & 18 & 8 & 79 & 0 & 100 \\
2 & 124 & 141 & 14 & 3 & 124 & 0 & 100 \\
3 & 169 & 197 & 13 & 15 & 169 & 0 & 100 \\
4 & 159 & 195 & 28 & 8 & 155 & 6 & 97.4 \\
5 & 174 & 203 & 17 & 12 & 174 & 0 & 100 \\
6 & 108 & 119 & 11 & 0 & 108 & 0 & 100 \\
7 & 112 & 122 & 6 & 4 & 112 & 0 & 100 \\
8 & 110 & 139 & 20 & 9 & 110 & 0 & 100 \\
9 & 215 & 253 & 33 & 5 & 215 & 0 & 100 \\
10 & 167 & 190 & 18 & 5 & 167 & 0 & 100 \\
\hline \multicolumn{7}{c}{ Average } \\
\hline
\end{tabular}

Figure 4 contains an example of the results of the image segmentation of the correct combination of watershed. Figure 4 point $\mathrm{A}$ is the result of watershed segmentation in the form of background segments, hole segments and score segments. Figure 4 point B 


\section{International Journal of Applied Sciences and Smart Technologies}

Volume 1, Issue 1, pages 51-64

ISSN 2655-8564

is an example of the results of a background segment and a hole segment is not an object so that it will experience a reduction process not an object. Figure 4 point $\mathrm{C}$ is an example of 2 segments which are low tones so that they will experience a process of combining objects. Figure 4 point $\mathrm{D}$ is an example of segmentation results that have taken the watershed combination process and the three segments are the correct segments.

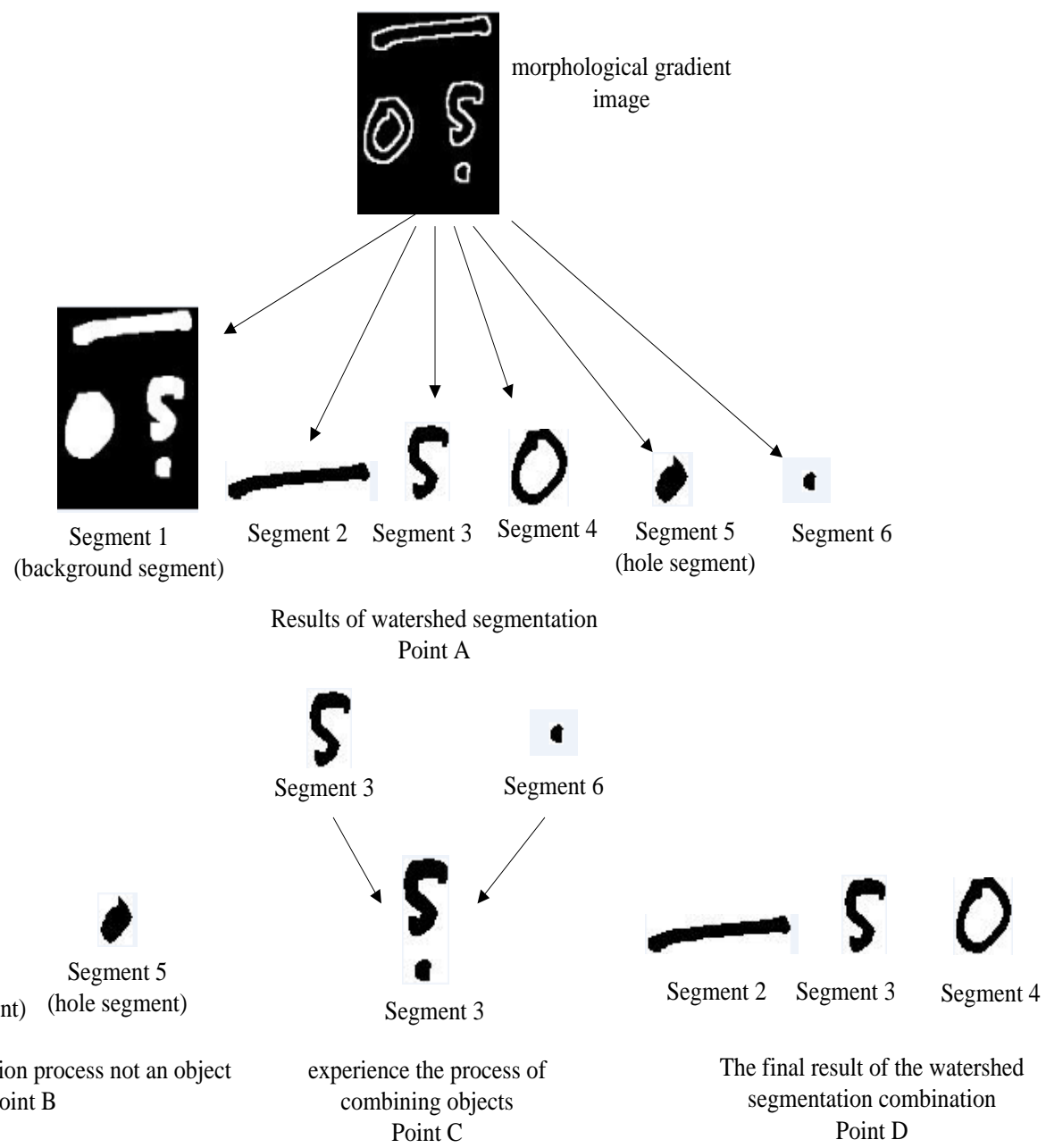

Figure 4. Examples of combined image results in combination watershed 


\section{International Journal of Applied Sciences and Smart Technologies}

Volume 1, Issue 1, pages 51-64

ISSN 2655-8564

\subsection{Results of Segment Accuracy in Watershed Segmentation without Combination}

In Table 3, the accuracy of $94.82 \%$ is obtained for the case of watershed segmentation without combination. The factor that causes decreased accuracy is the process like Figure 5. In Figure 5 point $A$ is the result of watershed segmentation without combination. Figure 5 point $\mathrm{B}$ is the remainder of the non-object segment, namely the background segment and the hole segment does not undergo a reduction process. Figure 5 point $\mathrm{C}$ segment 3 and segment 5 do not experience the process of combining objects into low notes. Figure 5 point $\mathrm{D}$ is the final result of watershed segmentation without combination.

Table 3. Result of segment accuracy in watershed segmentation without combination

\begin{tabular}{cccccc}
\hline \multirow{2}{*}{$\begin{array}{c}\text { Feature } \\
\text { image }\end{array}$} & $\begin{array}{c}\text { Original } \\
\text { image }\end{array}$ & $\begin{array}{c}\text { Segmentation } \\
\text { results image }\end{array}$ & True & False & $\begin{array}{c}\text { Accuracy } \\
(\%)\end{array}$ \\
\hline 1 & 79 & 102 & 71 & 31 & 89.87 \\
2 & 124 & 136 & 121 & 15 & 97.58 \\
3 & 169 & 194 & 154 & 40 & 91.12 \\
4 & 159 & 190 & 148 & 42 & 93.82 \\
5 & 174 & 198 & 162 & 36 & 93.1 \\
6 & 108 & 118 & 108 & 10 & 100 \\
7 & 112 & 119 & 108 & 11 & 96.43 \\
8 & 110 & 134 & 101 & 33 & 91.82 \\
9 & 215 & 248 & 211 & 37 & 98.14 \\
10 & 167 & 186 & 162 & 24 & 97.01 \\
\hline \multicolumn{5}{c}{ Average } \\
\hline
\end{tabular}




\section{International Journal of Applied Sciences and Smart Technologies}

Volume 1, Issue 1, pages 51-64

ISSN 2655-8564

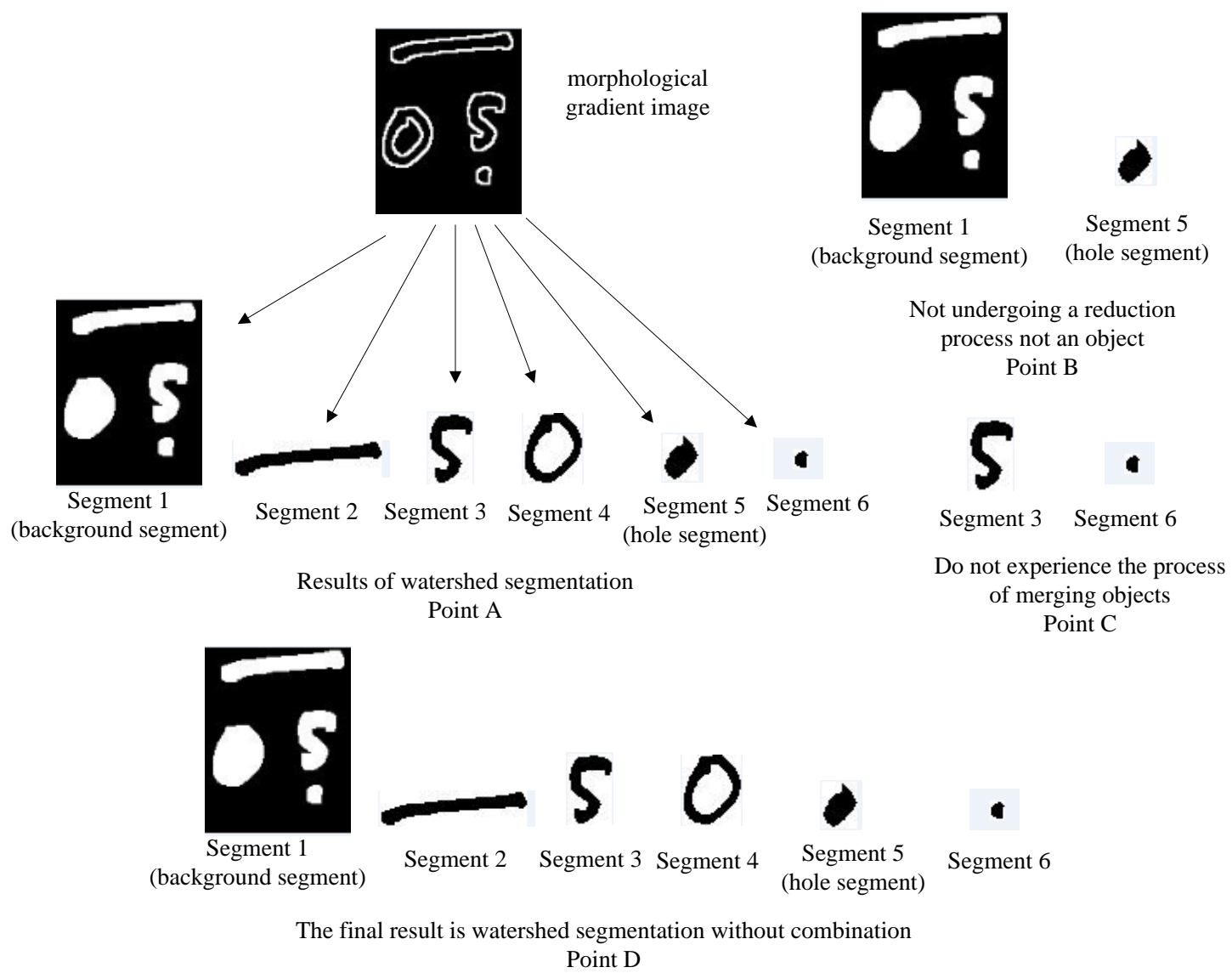

Figure 5. Examples of watershed image segmentation results without combination

\section{Conclusions}

Based on the results of research from system testing it can be concluded that the level of accuracy of segments in combination watershed segmentation is $99.74 \%$ higher than the level of accuracy of watershed segmentation without a combination of $94.82 \%$. Increased accuracy of $4.92 \%$ indicates that the combination watershed segmentation algorithm is better when compared to the watershed segmentation algorithm without combination. So that the combination of watershed segmentation algorithms can be used in the object recognition process.

\section{References}

[1] S. Wijayanti, Seni Budaya (Musik) Kelas X SMA Negeri 1 Pati, Seni Budaya Kelas X SMA, p. 2006, (2006). 


\section{International Journal of Applied Sciences and Smart Technologies}

Volume 1, Issue 1, pages 51-64

ISSN 2655-8564

[2] Murinto and A. Harjoko, "Segmentasi citra menggunakan watershed dan itensitas filtering sebagai pre processing," Seminar Nasional Informatika, 43-47, Mei 2009.

[3] D. Rahmawati, A. Harjoko, and R. Sumiharto, "Purwarupa sistem tracking sungai menggunakan unmanned aerial vehicle," Indonesian Journal of Electronics and Instrumentation Systems, 3 (2), 157-164, 2013.

[4] A. R. Himamunanto and A. R. Widiarti, "Javanese character image segmentation of document image of Hamong Tani," Digital Heritage International Congress, 1, 641-644, 2013.

[5] A. R. Widiarti, A. Harjoko, S. Hartati, and Marsono, "Implementasi model segmentasi manuskrip beraksara Jawa pada manuskrip beraksara Batak," Proceeding Seminar Nasional Inovasi dan Teknologi Informasi, 81-84, Oktober 2014.

[6] T. Shreekanth and V. Udayashankara, "A two stage braille character segmentation approach for embossed double sided Hindi devanagari braille documents," International Conference on Contemporary Computing and Informatics, 533-538, November 2014.

[7] S. Biswas and A. K. Das, "Text segmentation from scanned land map images using radon transform based projection profile," Proceedings of the International Conference of Soft Computing and Pattern Recognition (SoCPaR), 413-418, 2011.

[8] D. Min, "Research on numbered musical notation recognition and performance in a intelligent system," Institute of Information and Engineering Hunan University of Science and Engineering, 2-5, 2011.

[9] R. C. Gonzalez and R. E. Woods, "Digital Image Processing: 2nd," Publishing Company, USA, 2002.

[10]Y. Zhang and X. Cheng, "Medical image segmentation based on watershed and graph theory," no. 1, 1419-1422, 2010.

[11]K. Pinaryanto and A. R. Widiarti, "Implementasi segmentasi citra dokumen teks sastra Jawa menggunakan algoritma watershed," Undergraduate thesis, Universitas Sanata Dharma, 2009. 
International Journal of Applied Sciences and Smart Technologies

Volume 1, Issue 1, pages 51-64

ISSN 2655-8564

This page intentionally left blank 